\title{
PD-1 Inhibitors in the Advanced Esophageal Cancer
}

\author{
Ye Hong and Zhen-Yu Ding * \\ Department of Biotherapy, Cancer Center, West China Hospital, West China Medical School, Sichuan University, \\ Chengdu, China
}

OPEN ACCESS

Edited by:

Shuang Zhou,

University of Houston,

United States

Reviewed by:

Guobin Xia,

Baylor College of Medicine,

United States

Ka Wai Leong,

Dana-Farber Cancer Institute, United States

*Correspondence:

Zhen-Yu Ding

dingzhenyu@scu.edu.cn

Specialty section:

This article was submitted to

Pharmaceutical Medicine

and Outcomes Research,

a section of the journal

Frontiers in Pharmacology

Received: 29 June 2019 Accepted: 07 November 2019 Published: 29 November 2019

Citation:

Hong Y and Ding Z-Y (2019) $P D-1$ Inhibitors in the Advanced Esophageal Cancer.

Front. Pharmacol. 10:1418. doi: 10.3389/fphar.2019.01418
Esophageal cancer (EC) is a lethal disease, and ranks 7th in incidence and 6th in mortality worldwide. Patients are treated with surgery and/or chemoradiotherapy for a curative intent, but for those with advanced diseases systemic chemotherapy and targeted therapy are the mainstay treatment with poor prognosis. For the patients with squamous cell carcinoma and those progressed after chemotherapy, treatment option is even fewer, and effective treatment modalities are urgently needed. Preclinical and clinical studies have found the PD-1/PD-L1 inhibitors activate T lymphocytes, inhibit cancer growth, and improve survival in cancer patients. Multiple PD-1/PD-L1 inhibitors have been approved for the management of a variety of cancers. Interestingly, a large of proportion of EC patients have tumors with PD-L1 expression and high tumor mutation burden. Trials have been performed to evaluate the efficacy and safety of the PD-1/PD-L1 inhibitors in EC patients. This review will summarize the current progress in this field, especially the toxicities associated with these agents.

Keywords: esophageal carcinoma, gastroesophageal junction adenocarcinoma, PD-1 inhibitor, efficacy, safety

\section{INTRODUCTION}

Esophageal cancer (EC) is a dismal disease, with an estimated 5-year survival rate of only $20 \%$. Histologically, this disease entity is categorized to squamous cell carcinoma (SCC) and adenocarcinoma. In 2018, 572,034 new cases and 508,585 deaths were reported worldwide (Bray et al., 2018). Either surgery alone or with peri-operative chemotherapy is a curative treatment modality for locally advanced stage. For those in their late stages, systemic chemotherapy and targeted therapy are the mainstay treatment (Abdo et al., 2017). Platinum-based chemotherapy regimens, commonly combined with fluoropyrimidine or taxane, are the main treatment, with disappointing objective response rate (ORR) of $23.2 \%$ to $60.6 \%$, high incidence of adverse event, and a short overall survival (OS) of 7.7 to 15.5 months. And for the SCC patients and those progressed during or after chemotherapy, the treatment options are more limited. Single-agent chemotherapy, such as paclitaxel, docetaxel, and irinotecan, was recommended, resulting in an ORR of $20 \%$ and poor OS of approximately 5 months (Shi et al., 2013; Wang et al., 2013; Shirakawa et al, 2014; Prithviraj et al., 2015; Liu et al., 2016; Hiramoto et al., 2018). In summary, the existing treatments for EC have a limited efficacy and severe adverse events. Effective treatment modalities with moderate adverse event are urgently needed (Thallinger et al., 2011).

Lines of direct and indirect evidence show that the interaction between PD-1 and PD-L1 inhibits the function of $\mathrm{T}$ lymphocytes to evade persistent inflammatory or autoimmune reaction. However, this protective mechanism is hijacked by the tumors to escape the immune surveillance through upregulating PD-L1 expression on tumor cells (Mcdermott and Atkins, 2013; Araki et al., 2014; Guillebon et al., 2015; 
Chen and Han, 2015). Preclinical and clinical studies have found the PD-1/PD-L1 inhibitors activate T lymphocytes. And activated T lymphocytes help to inhibit cancer growth, and improve survival in cancer patients. PD-1/PD-L1 inhibitors have been approved for the management of a variety cancers, such as melanoma, lung cancer, and renal cell cancer etc. (Weber et al., 2015; Chedgy and Black, 2016; Reck et al., 2016). The efficiency of the PD-1/PD-L1 inhibitors is related to the PD-L1 expression, and/or tumor mutation burden (TMB) in tumor cells (Topalian et al., 2012; Rosenberg et al., 2016; Yarchoan et al., 2017; Hellmann et al., 2018a; Hellmann et al., 2018b; Hellmann et al., 2018c; Rizvi et al., 2018; Keenan et al., 2019). Interestingly, a large proportion of EC patients have tumors with PD-L1 expression (14.5-82.8\%, in different reports) and high TMB (Lawrence et al., 2013; Hsieh et al., 2018). Not surprisingly, trials have been initiated to evaluate the efficacy and safety of the PD-1/PD-L1 inhibitors in EC patients.

To this end, four antibodies (pembrolizumab, nivolumab, toripalimab, and camrelizumab) were tested in EC patients. Pembrolizumab and nivolumab are authorized globally for a dozen of cancers, including non-small cell lung cancer, head and neck squamous cell carcinoma, urothelial carcinoma, and so on. Toripalimab and camrelizumab are available in China with the indication for melanoma and classical Hodgkin lymphoma, respectively. Pembrolizumab and nivolumab had similar pharmacokinetic parameters. But no published data on that of toripalimab and camrelizumab are available now. Up to now, no clinical trial to directly compare these antibodies regarding safety and tolerability was reported. One report inferred pembrolizumab and nivolumab had similar safety profile (Wang et al., 2019). Data on direct comparison of clinical efficacy for these antibodies are lacking. This review provided a brief summary of current progress of these antibodies in the field of EC treatment, especially the toxicities associated with these agents.

\section{Data Acquisition}

The electronic database including PubMed, Clinical trials (https://clinicaltrials.gov/), Embase, Web of science, Cochrane library were retrieved by using the Keywords "esophageal cancer," "esophageal carcinoma," "immunotherapy," "PD-1," "PDL1," "clinical trial." The literature in abstract form was viewed, and those with only protocol design or preliminary results were excluded. Finally, 12 studies involving PD-1 inhibitor monotherapy with full description of the outcome were selected.

\section{Pembrolizumab}

Pembrolizumab is a humanized IgG4 antibody for PD-1. In a pilot phase $1 \mathrm{~b}$ study, KEYNOTE-012, pembrolizumab was first tested in patients with PD-L1-positive recurrent or metastatic gastroesophageal junction (GEJ) and gastric adenocarcinoma, without limitation on the number of lines of previous therapy (Muro et al., 2016). Thirty-nine patients were enrolled and received pembrolizumab $10 \mathrm{mg} / \mathrm{kg}$ every 2 weeks. The ORR was $22 \%$. Median progression-free survival (mPFS), median OS (mOS), and duration of response (DOR) were 1.9 months (mo), $11.4 \mathrm{mo}$, and 40 weeks. Treatment-related adverse events (TRAEs) occurred in $67 \%$ patients, grade 3 or 4 TRAEs in $13 \%$ patients $(\mathrm{n}=5)$. Fatigue $(18 \%)$, decreased appetite $(13 \%)$, hypothyroidism (13\%), pruritus (13\%), and arthralgia (10\%) were the most common TRAEs. Grade 3-4 TRAEs included grade 3 fatigue $(n=2)$, grade 3 pemphigoid $(n=1)$, grade 3 hypothyroidism $(n=1)$, grade 3 peripheral sensory neuropathy $(n=1)$, and grade 4 pneumonitis $(n=1)$. There were no treatment-related death or discontinuation of drugs due to TRAE.

Phase 2 trial KEYNOTE-059 investigated the efficacy and safety of pembrolizumab monotherapy in the late $(\geq 3)$ lines of therapy (Fuchs et al., 2018). Two hundred fifty-nine patients with similar features as those in KEYNOTE-012 study were enrolled, except for no requirement of PD-L1 expression. Pembrolizumab was given every 3 weeks (at fixed dose of $200 \mathrm{mg}$ ). The ORR for the intention-to-treatment (ITT) cohort was $11.6 \%$, and in PD-L1-positive and -negative cohorts, it was $15.5 \%$ and $6.4 \%$, respectively. The $\mathrm{mDOR}$ for ITT, $\mathrm{PD}-\mathrm{L} 1$-positive, and $\mathrm{PD}-\mathrm{L} 1-$ negative patients was $8.4 \mathrm{mo}, 16.3 \mathrm{mo}$, and $6.9 \mathrm{mo}$, respectively. The mPFS and mOS of ITT patients were 2 mo and $5.6 \mathrm{mo}$. TRAEs of any grade and grade $3-5$ occurred in $60.2 \%$ and $17.8 \%$ patients. Fatigue, pruritus, rash, hypothyroidism, decreased appetite, anemia, nausea, diarrhea, and arthralgia were the most common TRAEs. There were two treatment-related deaths and two cases of treatment-related discontinuation.

The efficacy of pembrolizumab in the second-line therapy was tested in a randomized controlled phase 3 trial KEYNOTE-061 (Shitara et al., 2018). Five hundred ninety-two patients with advanced GEJ or gastric adenocarcinoma who progressed after chemotherapy regimen of fluoropyrimidine and platinum were enrolled. Pembrolizumab (200 mg) every 3 weeks for up to 2 years or paclitaxel $80 \mathrm{mg} / \mathrm{m}^{2}$ on days $1,8,15$ in a 4 -week cycle was administered. In population with $\mathrm{PD}-\mathrm{L} 1 \geq 1 \%$ (PD-L1 CPS $\geq 1$ ), the mOS of pembrolizumab and chemotherapy was 9.1 and $1.5 \mathrm{mo}$. And mPFS was 8.3 and $4.1 \mathrm{mo}$, respectively. The ORR of pembrolizumab and chemotherapy was $16 \%$ and $14 \%$, and mDOR was 18 and $5.2 \mathrm{mo}$. In the ITT population, TRAEs occurred in $53 \%$ and $84 \%$ patients receiving pembrolizumab and chemotherapy, and for grade 3-5 TRAEs, the incidence was $14 \%$ and $35 \%$. The most common grade 3-5 TRAE for pembrolizumab were anemia and fatigue. Three percent of the patients in pembrolizumab group discontinued treatment because of TRAEs. The mortality rate was $1 \%$ in pembrolizumab group.

KEYNOTE-062 was a phase 3 trial to investigate pembrolizumab with $(p+c)$ or without $(p)$ chemotherapy versus chemotherapy (c, cisplatin, and fluoropyrimidine) for the first-line treatment (Tabernero et al., 2019). This study was also conducted in the GEJ and gastric adenocarcinoma with PD-L1 CPS $\geq 1$. Totally, 763 patients were enrolled. Pembrolizumab monotherapy compared with chemotherapy did not show any survival benefit. In patients with $\mathrm{PD}$-L1 CPS $\geq 10$, pembrolizumab monotherapy showed improved mOS over chemotherapy (17.4 and $10.8 \mathrm{mo}$ ), but inferior mPFS (2.9 and $6.1 \mathrm{mo}$ ) and ORR (25\% and 36.7\%). P+c vs c did not show any benefit in OS and PFS regardless of patients PD-L1 CPS status (CPS $\geq 1$ or $\mathrm{CPS} \geq 10$ ). Grade 3-5 TRAE rates were $17 \%(\mathrm{p})$, $73 \%(\mathrm{p}+\mathrm{c})$, and $65 \%(\mathrm{c})$.

Pembrolizumab was also tested in other histological types, mainly SCC. KEYNOTE-028 was a phase 1 b study similar to KEYNOTE-012 study, to explore the efficacy and safety of 
pembrolizumab in late-line treatment ( $87 \%$ patients had received $\geq 2$ lines of treatment) for all histological types (including SCC, adenocarcinoma, (Doi et al., 2018). Twenty-three patients with PD-L1-positive tumors were enrolled. The incidence rate of TRAEs was 39\%, and the mOS was 7 months.

A phase 2 trial KEYNOTE-180 was similar to KEYNOTE-059 study, investigating pembrolizumab monotherapy in the setting of late ( $\geq 3$ ) lines of therapy (Shah et al., 2019). But this study recruited patients of all histological types. PD-L1(+) was mandatory, defined as CPS $\geq 10$. One hundred twentyone patients were enrolled. The ORR was $14.3 \%$ among SCC patients, and 5.2\% among adenocarcinoma patients. The mPFS and mOS were 2 and 5.8 mo. Subgroup analysis showed mOS was better in patients with SCC. The incidence rates of TRAEs and grade $3-5$ TRAEs were $57.9 \%$ and $12.4 \%$, respectively. The most commonly TRAEs included fatigue, rash, pruritus, hypothyroidism, and diarrhea. Treatment-related discontinuation $(n=5)$ and death $(n=1)$ were reported.

KEYNOTE-181 was a phase 3 trial similar to KEYNOTE-061, where pembrolizumab was used in the second line of therapy, except for recruitment of all histotypes (Kojima et al., 2019). Six hundred twenty-eight patients were enrolled. In the ITT population, pembrolizumab compared with chemotherapy did not show significant benefit in mOS and mPFS. But in subgroup of patients with $\mathrm{PD}-\mathrm{L} 1 \mathrm{CPS} \geq 10$, pembrolizumab treatment led to longer mOS over chemotherapy (9.3 and $6.7 \mathrm{mo}$ ) with statistical significance. The ORR was also improved $(21.5 \%$ and $6.1 \%$ ) in this subpopulation. The incidence rate of TRAEs of pembrolizumab and chemotherapy were $64.3 \%$ and $86.1 \%$. The incidence rates of grade 3-5 TRAEs were $18.2 \%$ and $40.7 \%$, respectively. There was no significant difference in treatmentrelated discontinuation (6.1\% vs $6.4 \%$ ) and death (1.5\% vs $1.7 \%)$.

\section{Nivolumab}

Nivolumab is another humanized IgG4 monoclonal antibody for PD-1 immune checkpoint. For patients with advanced GEJ and gastric adenocarcinoma, nivolumab was tested in trial ATTRACTION-2 (in Asia) and CheckMate-032 (in Western countries).

ATTRACTION-2 was a randomized, double-blind, placebocontrolled, phase 3 trial to evaluate the efficacy and safety of nivolumab in heavily pretreated adenocarcinoma (Kang et al., 2017). Four hundred ninety-three patients were enrolled and were randomly assigned $(2: 1)$ to receive nivolumab $3 \mathrm{mg} / \mathrm{kg}$ or placebo every 2 weeks. The mOS of nivolumab and placebo was 5.26 and $4.14 \mathrm{mo}$, and the MPFS was 1.61 and $1.45 \mathrm{mo}$. The ORR and mDOR of nivolumab were $11.2 \%$ and 9.53 mo. The incidence of TRAEs and grade 3-5 TRAEs of nivolumab was $43 \%$ and $10 \%$. The common TRAEs included pruritus, diarrhea, rash, and fatigue. In the nivolumab group, nine cases of treatment discontinuation and five deaths occurred.

Conducted in a cohort with similar demographic features, CheckMate-032 was a phase $1 / 2$ trial where nivolumab monotherapy or nivolumab plus ipilimumab were administered (Janjigian et al., 2018). One hundred sixty patients were enrolled. The treatment consisted three arms: either nivolumab
$3 \mathrm{mg} / \mathrm{kg}$ every 2 weeks $(\mathrm{n}=59)$, or nivolumab $1 \mathrm{mg} / \mathrm{kg}$ plus ipilimumab $3 \mathrm{mg} / \mathrm{kg}$ every 3 weeks for four cycles $(\mathrm{N} 1 \mathrm{I} 3, \mathrm{n}=$ 49), or nivolumab $3 \mathrm{mg} / \mathrm{kg}$ plus ipilimumab $1 \mathrm{mg} / \mathrm{kg}$ every 3 weeks for four cycles (N3I1, n = 52). After four cycles, all patients were maintained on nivolumab $3 \mathrm{mg} / \mathrm{kg}$ therapy. For the group of nivolumab monotherapy, N1I3, and N3I1, the ORR was $12 \%, 24 \%$ and $8 \%$. The mOS was $6.2,6.9,4.8 \mathrm{mo}$, and the mPFS was $1.4,1.4$, and $1.6 \mathrm{mo}$. The incidence rates of TRAEs and grade 3-4 TRAEs of these groups were $69 \%, 17 \%$, $84 \%$, and $47 \%, 75 \%, 27 \%$, respectively. The most frequently occurred TRAEs were fatigue, pruritus, rash, diarrhea, decreased appetite, and increased transaminase. The incidence rates of discontinuation of drug related to TRAEs were $3 \%$, $20 \%$, and $13 \%$ in three groups.

Another phase 2 study ATTRACTION-1 was conducted in Japan, where patients with EC were enrolled (Kudo et al., 2017). Sixty-five patients with heavily treated SCC were enrolled and received nivolumab $3 \mathrm{mg} / \mathrm{kg}$ every 2 weeks. The ORR was $17 \%$, mOS was 10.8 months, and mPFS was 1.5 months. The incidence rates of TRAEs and grade 3 or worse TRAEs were $60 \%$ and $17 \%$, respectively. The most common adverse events were diarrhea, decreased appetite, constipation, rash, and fatigue. Seven patients discontinued therapy due to TRAEs, and no death related to TRAE occurred.

\section{Toripalimab (JS001) and Camrelizumab (SHR1210)}

Toripalimab and camrelizumab are two of Chinese domestic me-too antibodies in this class. A phase $1 \mathrm{~b} / 2$ trial (Clinicaltrial identifier: NCT02915432) evaluated the efficacy and safety of toripalimab in refractory/metastatic esophageal SCC (Xu et al., 2018). Fifty-six patients were enrolled and received toripalimab at the dose of $3 \mathrm{mg} / \mathrm{kg}$ every 2 weeks. Till September 2017, 34 patients were evaluated, and 8 patients achieved partial response with an ORR of $23.5 \%$. TRAEs were mostly grade 1 or 2. Another trial (NCT02742935) was a dose-escalating phase 1 study investigating the efficacy and safety of camrelizumab in $\geq 2$ line treatment of esophageal SCC (Huang et al., 2018). The dose was given at 60,200, and $400 \mathrm{mg}$ every 2 weeks. The ORR was $33.3 \%$ and the mPFS was 3.6 months. The incidences of TRAEs and grade 3 TRAEs were $83.3 \%$ and $10 \%$, respectively. The most common TRAEs included reactive capillary hemangiomas, pruritus, hypothyroidism, and fever. There was no treatmentrelated discontinuation due to toxicity.

\section{DISCUSSION}

EC is a lethal disease affecting millions of people worldwide. Histologically, it is composed of two main subtypes, i.e., SCC and adenocarcinoma. They differ to a large extent in their genetic aberrations, epidemiology, etiology, and clinical manifestations. Thus, the two subtypes should have distinct strategy of therapy. Previously radio- and chemo-therapy remain the mainstay of the therapy for those unsuitable for surgery. Targeted therapy including anti-angiogenesis agents and epidermal growth factor receptor inhibitors 
obtains authorization for the treatment of adenocarcinoma, but not SCC. Therefore, there is a large unmet need for the improvement of SCC treatment. It is in high expectance that the immune checkpoint inhibitors help to advancing the progress in this field. Our summary showed that most of the studies were performed in adenocarcinoma till now, but the trends toward SCC became obvious (Figure 1).

The current review summarized 12 trials on PD-1 inhibitor monotherapy for the treatment of advanced EC, including phase $3(n=4)$ and phase $1 / 2$ trials $(n=8)$. Among them, KEYNOTE-062 is the only one investigating pembrolizumab monotherapy in the first-line treatment. Both KEYNOTE-061 and KEYNOTE-181 investigated the efficacy of pembrolizumab monotherapy in the second-line treatment. The rest nine trials investigated efficacy and safety of PD-1 inhibitors in late lines. The immune checkpoint inhibitors showed promising results, with minimal to mild toxicities (Figure 2). TRAEs in EC were similar to those reported in other solid tumors, and no unexpected TRAEs occurred (Topalian et al., 2012; Garon et al., 2015; Ferris et al., 2016; Tomita et al., 2017).

It was interesting to compare the PD-1/PD-L1 inhibitors and traditional chemotherapy for EC patients. The two modalities were compared in a head-to-head fashion in three of the trials (Table 1). Although relative a small sample, a clear trend could be easily found favoring the former, with elevated ORRs, prolonged PFS and OS, and less frequency of any-grade or grade 3-5 AE in immunotherapy.

Pembrolizumab, nivolumab, and toripalimab had similar incidence of TRAE (Figure 2, 39\%-67\%, 43\%-60\%, 62.7\%, respectively), lower than that of camrelizumab (83.3\%). Also, grade 3-5 TRAEs seemed less likely in pembrolizumab
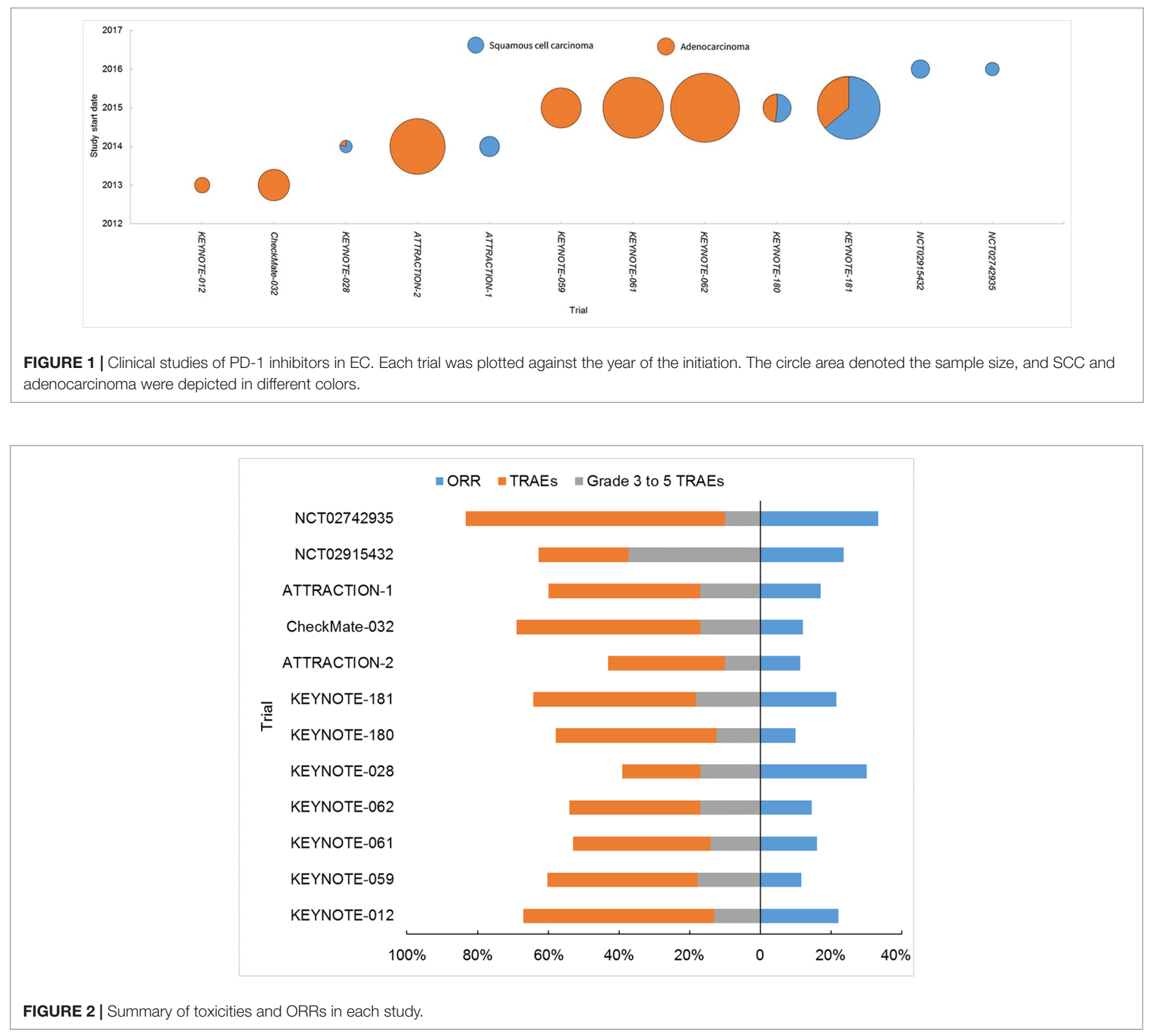
TABLE 1 | The comparison of PD-1 inhibitors and chemotherapy.

\begin{tabular}{|c|c|c|c|c|c|c|c|c|}
\hline Trial & Drug & PD-L1 CPS & ORR (\%) & DOR (mo) & PFS (mo) & OS (mo) & AEs (\%) & AE $\geq$ Grade $3(\%)$ \\
\hline & & $<1$ & 2 vs 10.4 & NA & NA & 4.8 vs 8.2 & & \\
\hline \multirow[t]{2}{*}{ KEYNOTE-061 } & Pembro vs Chemo & $\geq 1$ & 16 vs 14 & 8 vs 5.2 & 1.5 vs 4.1 & 9.1 vs 8.3 & 53 vs 84 & 14 vs 35 \\
\hline & & $\geq 10$ & 24.5 vs 9.1 & NA & NA & 10.4 vs 8 & & \\
\hline \multirow[t]{2}{*}{ KEYNOTE-062 } & Pembro vs Chemo & $\geq 1$ & 14.5 vs 36.8 & NA & 2 vs 6.4 & 10.6 vs 11.1 & 54.3 vs 91.8 & 17 vs 69 \\
\hline & & $\geq 10$ & 25 vs 36.7 & NA & 2.9 vs 6.1 & 17.4 vs 10.8 & & \\
\hline \multirow[t]{2}{*}{ KEYNOTE-181 } & Pembro vs Chemo & All comer & 13.1 vs 6.7 & 8.5 vs 10.7 & 2.1 vs 3.4 & 7.1 vs 7.1 & 64.3 vs 86.1 & 18.2 vs 40.9 \\
\hline & & $\geq 10$ & 21.5 vs 6.1 & 9.3 vs 7.7 & 2.6 vs 3 & 9.3 vs 6.7 & & \\
\hline
\end{tabular}

TABLE 2 | Ongoing phase 2/3 trial with PD-1 inhibitor combined with chemotherapy in first-line treatment of esophageal carcinoma.

\begin{tabular}{|c|c|c|c|c|c|}
\hline Trial & Phase & Status & Drug & Tumor & Treatment \\
\hline $\begin{array}{l}\text { KEYNOTE-590-China } \\
\text { Extension Study }\end{array}$ & 3 & Recruiting & Pembrolizumab+Cisplatin+5-FU/Placebo+Cisplatin+5-FU & Esophageal Carcinoma & First-line \\
\hline KEYNOTE-590 & 3 & Active & Pembrolizumab+Cisplatin+5-FU/Placebo+Cisplatin+5-FU & Esophageal Carcinoma & First-line \\
\hline NCT02954536 & 2 & Recruiting & $\begin{array}{l}\text { Pembrolizumab +Trastuzumab+ Chemotherapy } \\
\text { (Capecitabine/5-Fluorouracil+Cisplatin/Oxaliplatin) }\end{array}$ & Esophagogastric Carcinoma & First-line \\
\hline NCT03342937 & 2 & Recruiting & Pembrolizumab + Oxaliplatin +Capecitabine & Esophagogastric Carcinoma & First-line \\
\hline NCT03615326 & 3 & Recruiting & $\begin{array}{l}\text { Pembrolizumab+Trastuzumab+Chemotherapy/Placebo+Trastuzu } \\
\text { mab+Chemotherapy (Capecitabine/5-Fluorouracil/S-1+Cisplatin/ } \\
\text { Oxaliplatin) }\end{array}$ & $\begin{array}{l}\text { Gastroesophageal junction and gastric } \\
\text { adenocarcinoma }\end{array}$ & First-line \\
\hline Checkmate 648 & 3 & Recruiting & $\begin{array}{l}\text { Nivolumab + Ipilimumab/Nivolumab + Cisplatin + Fluorouracil/ } \\
\text { Cisplatin + Fluorouracil }\end{array}$ & Esophageal Carcinoma & First-line \\
\hline NCT03409848 & 3 & Recruiting & Nivolumab and Trastuzumab +|pilimumab/FOLFOX & Esophagogastric Carcinoma & First-line \\
\hline NCT03829969 & 3 & Recruiting & JS001 +paclitaxel +cisplatin/placebo +paclitaxel +cisplatin & Esophageal Squamous Cell Carcinoma & First-line \\
\hline NCT03691090 & 3 & Recruiting & SHR-1210 + paclitaxel + cisplatin/placebo +paclitaxel +cisplatin & Esophageal squamous cell carcinoma & First-line \\
\hline NCT03603756 & 2 & Recruiting & SHR-1210 + Apatinib+ Chemotherapy (irinotecan/paclitaxel+ nedaplatin) & Esophageal Squamous Cell Carcinoma & First-line \\
\hline
\end{tabular}

(12.4\%-18.2\%), nivolumab (10\%-17\%), camrelizumab (10\%) than toripalimab $(37.3 \%)$. The incidence of immune-related adverse events (irAEs) was $18 \%$ to $26 \%$ in pembrolizumab, $10.2 \%$ in toripalimab, and $83.3 \%$ in camrelizumab. It should be noted the toxicities of toripalimab and camrelizumab were both extracted from small-sized, phase 1 studies, and might be over-estimated.

Next, specific AE was analyzed. Because the information was lacking for toripalimab and camrelizumab, only pembrolizumab and nivolumab were compared. A consistent higher incidence was observed in hyperthyroidism $(3.5 \%-7.7 \%$ and $1 \%)$, hypothyroidism $(7.4 \%-12.8 \%$ and 0$)$, pneumonitis $(1.9 \%-4.9 \%$ and $0.3 \%)$, colitis $(1 \%-2.6 \%$ and $1 \%)$, and hepatitis $(0.4 \%-2.6 \%$ and 0$)$ for pembrolizumab than nivolumab. For the severe (grade 3-5) irAE, pembrolizumab also had worse record in hypothyroidism $(0.4 \%-2.5 \%$ and 0$)$, pneumonitis $(0.3 \%-2.6 \%$ and $0.3 \%)$, colitis $(0.3 \%-1.2 \%$ and $0.3 \%)$, and hepatitis $(0.4 \%-1 \%$ and 0$)$ than nivolumab. But it was imprudent to make direct comparison of data from different trials. For EC treatment, these four agents had comparable safety and efficiency, based on the direct comparison of their reported outcomes (Figure 2). This conclusion also got supports from the biochemical features of these drugs. They are monoclonal antibodies blocking $\mathrm{PD}-1$, and they have the same, if any difference, of action mechanism.

From these trials, one reasons that PD-1 inhibitors would play a role in the treatment of advanced EC. But the question is when and how to apply these agents appropriately. At this time point, Food and Drug Administration (FDA) authorized pembrolizumab for the late $(\geq 2)$ line treatment for the cancer patients whose tumors harbor high TMB, irrespective of tissue origin, also including those with EC. Additionally, FDA approved pembrolizumab for the 2-line treatment for the patients with SCC with CPS $\geq 10$ and for the 3-line treatment for the patients of with GEJ and gastric adenocarcinoma with $\mathrm{PD}-\mathrm{L} 1 \mathrm{CPS} \geq 1$. Based on the encouraging results, PD-1 inhibitor combined with chemotherapy for the first-line therapy for EC is in underway (Table 2).

\section{CONCLUSION}

In general, PD-L1 inhibitor monotherapy in the treatment of pretreated EC has a promising antitumor activity and manageable toxicity.

\section{AUTHOR CONTRIBUTIONS}

Z-YD and YH contributed conception and overall idea of the study. YH wrote the first draft of the manuscript. Z-YD wrote sections of the manuscript. Both authors contributed to manuscript revision, read and approved the submitted version. 


\section{REFERENCES}

Abdo, J., Agrawal, D. K., and Mittal, S. K. (2017). Basis for molecular diagnostics and immunotherapy for esophageal cancer. Expert Rev. Anticancer Ther. 17 (1), 33-45. doi: 10.1080/14737140.2017.1260449

Araki, K., Youngblood, B., and Ahmed, R. (2014). Programmed Cell Death 1-Directed Immunotherapy for Enhancing T-Cell Function. Cold Spring Harb Symp. Quant. Biol. 78 (1), 239-247. doi: 10.1101/sqb.78.019869

Bray, F., Ferlay, J., Soerjomataram, I., Siegel, R. L., Torre, L. A., and Jemal, A. (2018). Global cancer statistics 2018: GLOBOCAN estimates of incidence and mortality worldwide for 36 cancers in 185 countries. CA: Cancer J. Clinicians. 68 (6), 394-424. doi: 10.3322/caac. 21492

Chedgy, E. C. P., and Black, P. C. (2016). Nivolumab: the new second line treatment for advanced renal-cell carcinoma commentary on: nivolumab versus everolimus in advanced renal-cell carcinoma. Urology. 89, 8-9. doi: 10.1016/j. urology.2015.12.003

Chen, L., and Han, X. (2015). Anti-PD-1/PD-L1 therapy of human cancer: past, present, and future. J. Clin. Invest. 125 (9), 3384-3391. doi: 10.1172/ JCI80011

Doi, T., Piha-Paul, S. A., Jalal, S. I., Saraf, S., Lunceford, J., Koshiji, M., et al. (2018). Safety and antitumor activity of the anti-programmed death-1 antibody pembrolizumab in patients with advanced esophageal carcinoma. J. Clin. Oncol. 36 (1), 61-67. doi: 10.1200/jco.2017.74.9846

Ferris, R. L., Blumenschein, G., Fayette, J., Guigay, J., Colevas, A. D., Licitra, L., et al. (2016). Nivolumab for recurrent squamous-cell carcinoma of the head and neck. New Engl. J. Med. 375 (19), 1856-1867. doi: 10.1056/ NEJMoa1602252

Fuchs, C. S., Doi, T., Jang, R. W., Muro, K., Satoh, T., Machado, M., et al. (2018). Safety and efficacy of pembrolizumab monotherapy in patients with previously treated advanced gastric and gastroesophageal junction cancer: Phase 2 Clinical KEYNOTE-059 Trial. JAMA Oncol. 4 (5), e180013. doi: 10.1001/ jamaoncol.2018.0013

Garon, E. B., Rizvi, N. A., Hui, R., Leighl, N., Balmanoukian, A. S., Eder, J. P., et al. (2015). Pembrolizumab for the treatment of non-small-cell lung cancer. New Engl. J. Med. 372 (21), 2018-2028. doi: 10.1056/NEJMoa1501824

Guillebon, E. D., Roussille, P., Frouin, E., and Tougeron, D. (2015). Anti program death-1/anti program death-ligand 1 in digestive cancers. World J. Gastrointest Oncol. 7 (8), 95-101. doi: 10.4251/wjgo.v7.i8.95

Hellmann, M. D., Callahan, M. K., Awad, M. M., Calvo, E., Ascierto, P. A., Atmaca, A., et al. (2018a). Tumor mutational burden and efficacy of nivolumab monotherapy and in combination with ipilimumab in small-cell lung cancer. Cancer Cell 33 (5), 853-861. doi: 10.1016/j.ccell.2018.04.001

Hellmann, M. D., Ciuleanu, T. E., Pluzanski, A., Lee, J. S., Otterson, G. A., Audigier-Valette, C., et al. (2018b). Nivolumab plus ipilimumab in lung cancer with a high tumor mutational burden. New Engl. J. Med. 378 (22), 2093-2104. doi: 10.1056/NEJMoa1801946

Hellmann, M. D., Nathanson, T., Rizvi, H., Creelan, B. C., Sanchez-Vega, F., Ahuja, A., et al. (2018c). Genomic features of response to combination immunotherapy in patients with advanced non-small-cell lung cancer. Cancer Cell. 33 (5), 843852.e4. doi: 10.1016/j.ccell.2018.03.018

Hiramoto, S., Kato, K., Shoji, H., Okita, N., Takashima, A., Honma, Y., et al. (2018). A retrospective analysis of 5-fluorouracil plus cisplatin as first-line chemotherapy in the recent treatment strategy for patients with metastatic or recurrent esophageal squamous cell carcinoma. Int. J. Clin. Oncol. 23 (3), 466-472. doi: 10.1007/s10147-018-1239-x

Hsieh, C.-C., Hsu, H.-S., and Chen, Y.-J. (2018). AB018. Clinical relevance of PD-L1 and PD-L2 overexpression in patients with esophageal squamous cell carcinoma. J. Thoracic Dis. 9 (S14), AB018-AB018. doi: 10.21037/jtd.2017.s018

Huang, J., Xu, B., Mo, H., Zhang, W., Chen, X., Wu, D., et al. (2018). Safety, activity, and biomarkers of SHR-1210, an anti-PD-1 antibody, for patients with advanced esophageal carcinoma. Clin. Cancer Res. 24 (6), 1296-1304. doi: 10.1158/1078-0432.CCR-17-2439

Janjigian, Y. Y., Bendell, J., Calvo, E., Kim, J. W., Ascierto, P. A., Sharma, P., et al. (2018). CheckMate-032 study: efficacy and safety of nivolumab and nivolumab plus ipilimumab in patients with metastatic esophagogastric cancer. J. Clin. Oncol. 36 (28), 2836-2844. doi: 10.1200/jco.2017.76.6212

Kang, Y. K., Boku, N., Satoh, T., Ryu, M. H., Chao, Y., Kato, K., et al. (2017). Nivolumab in patients with advanced gastric or gastro-oesophageal junction cancer refractory to, or intolerant of, at least two previous chemotherapy regimens (ONO-4538-12, ATTRACTION-2): a randomised, double-blind, placebo-controlled, phase 3 trial. Lancet. 390 (10111), 2461-2471. doi: 10.1016/ S0140-6736(17)31827-5

Keenan, T. E., Burke, K. P., and Van Allen, E. M. (2019). Genomic correlates of response to immune checkpoint blockade. Nat. Med. 25 (3), 389-402. doi: 10.1038/s41591-019-0382-x

Kojima, T., Muro, K., Francois, E., Hsu, C.-H., Moriwaki, T., Kim, S.-B., et al. (2019). Pembrolizumab versus chemotherapy as second-line therapy for advanced esophageal cancer: The Phase 3 KEYNOTE-181 study. J. Clin. Oncol. 37 (4_suppl), 2-2. doi: 10.1200/JCO.2019.37.4_suppl.2

Kudo, T., Hamamoto, Y., Kato, K., Ura, T., Kojima, T., Tsushima, T., et al. (2017). Nivolumab treatment for oesophageal squamous-cell carcinoma: an openlabel, multicentre, phase 2 trial. Lancet Oncol. 18 (5), 631-639. doi: 10.1016/ S1470-2045(17)30181-X

Lawrence, M. S., Stojanov, P., Polak, P., Kryukov, G. V., Cibulskis, K., Sivachenko, A., et al. (2013). Mutational heterogeneity in cancer and the search for new cancerassociated genes. Nature 499, 214-218. doi: 10.1038/nature12213

Liu, Y., Ren, Z., Yuan, L., Xu, S., Yao, Z., Qiao, L., et al. (2016). Paclitaxel plus cisplatin vs. 5-fluorouracil plus cisplatin as first-line treatment for patients with advanced squamous cell esophageal cancer. Am. J. Cancer Res. 6 (10), 2345-2350.

Mcdermott, D. F., and Atkins, M. B. (2013). PD-1 as a potential target in cancer therapy. Cancer Med. 2 (5), 662-673. doi: 10.1002/cam4.106

Muro, K., Chung, H. C., Shankaran, V., Geva, R., Catenacci, D., Gupta, S., et al. (2016). Pembrolizumab for patients with PD-L1-positive advanced gastric cancer (KEYNOTE-012): a multicentre, open-label, phase $1 \mathrm{~b}$ trial. Lancet Oncol. 17 (6), 717-726. doi: 10.1016/S1470-2045(16)00175-3

Prithviraj, G.K., Baksh, K., Fulp, W., Meredith, K., Hoffe, S., Shridhar, R., et al. (2015). Carboplatin and paclitaxel as first-line treatment of unresectable or metastatic esophageal or gastric cancer. Dis. Esophagus. 28 (8), 782-787. doi: $10.1111 /$ dote. 12279

Reck, M., Rodríguez-Abreu, D., Robinson, A. G., Hui, R., Csőszi, T., Fülöp, A., et al. (2016). Pembrolizumab versus chemotherapy for PD-L1-Positive Nonsmall-cell lung cancer. New Engl. J. Med. 375 (19), 1823-1833. doi: 10.1056/ nejmoa1606774

Rizvi, H., Sanchez-Vega, F., La, K., Chatila, W., Jonsson, P., Halpenny, D., et al. (2018). Molecular determinants of response to anti-programmed cell death (PD)-1 and anti-programmed death-ligand 1 (PD-L1) blockade in patients with non-small-cell lung cancer profiled with targeted next-generation sequencing. J. Clin. Oncol.: Off. J. Am. Soc. Clin. Oncol. 36 (7), 633-641. doi: 10.1200/JCO.2017.75.3384

Rosenberg, J. E., Hoffman-Censits, J., Powles, T., Heijden, M. S. V. D., Balar, A. V., Necchi, A., et al. (2016). Atezolizumab in patients with locally advanced and metastatic urothelial carcinoma who have progressed following treatment with platinum-based chemotherapy: a single-arm, multicentre, phase 2 trial. Lancet 387 (10031), 1909-1920. doi: 10.1016/S0140-6736(16)00561-4

Shah, M. A., Kojima, T., Hochhauser, D., Enzinger, P., Raimbourg, J., Hollebecque, A., et al. (2019). Efficacy and safety of pembrolizumab for heavily pretreated patients with advanced, metastatic adenocarcinoma or squamous cell carcinoma of the esophagus: the phase 2 KEYNOTE-180 studypembrolizumab for heavily pretreated patients with advanced carcinoma of the esophaguspembrolizumab for heavily pretreated patients with advanced carcinoma of the esophagus. JAMA Oncol. 5 (4), 546-550. doi: 10.1001/ jamaoncol.2018.5441

Shi, Y., Qin, R., Wang, Z. K., and Dai, G. H. (2013). Nanoparticle albumin-bound paclitaxel combined with cisplatin as the first-line treatment for metastatic esophageal squamous cell carcinoma. Onco. Targets Ther. 6, 585-591. doi: 10.2147/OTT.S44406

Shirakawa, T., Kato, K., Nagashima, K., Nishikawa, A., Sawada, R., Takahashi, N., et al. (2014). A retrospective study of docetaxel or paclitaxel in patients with advanced or recurrent esophageal squamous cell carcinoma who previously received fluoropyrimidine- and platinumbased chemotherapy. Cancer Chemother. Pharmacol. 74 (6), 1207-15. doi: 10.1007/s00280-014-2597-3

Shitara, K., Özgüroğlu, M., Bang, Y. J., Di Bartolomeo, M., Mandalà, M., et al. (2018). Pembrolizumab versus paclitaxel for previously treated, advanced gastric or gastro-oesophageal junction cancer (KEYNOTE-061): a randomised, 
open-label, controlled, phase 3 trial. Lancet 392 (10142), 123-133. doi: 10.1016/ S0140-6736(18)31257-1

Tabernero, J., Cutsem, E. V., Bang, Y.-J., Fuchs, C. S., Wyrwicz, L., Lee, K. W., et al. (2019). Pembrolizumab with or without chemotherapy versus chemotherapy for advanced gastric or gastroesophageal junction (G/GEJ) adenocarcinoma: The phase III KEYNOTE-062 study. J. Clin. Oncol. 37 (18_suppl), LBA4007LBA4007. doi: 10.1200/JCO.2019.37.18

Thallinger, C. M. R., Raderer, M., and Hejna, M. (2011). Esophageal cancer: a critical evaluation of systemic second-line therapy. J. Clin. Oncol. 29 (35), 4709-4714. doi: 10.1200/jco.2011.36.7599

Tomita, Y., Fukasawa, S., Shinohara, N., Kitamura, H., Oya, M., Eto, M., et al. (2017). Nivolumab versus everolimus in advanced renal cell carcinoma: Japanese subgroup analysis from the CheckMate 025 study. Japanese J. Clin. Oncol. 47 (7), 639-646. doi: 10.1093/jjco/hyx049

Topalian, S. L., Hodi, F. S., Brahmer, J. R., Gettinger, S. N., Smith, D. C., Mcdermott, D. F., et al. (2012). Safety, activity, and immune correlates of anti-PD-1 antibody in cancer. New Engl. J. Med. 366 (26), 2443-2454. doi: 10.1056/NEJMoa1200690

Wang, J., Chang, J., Yu, H., Wu, X., Wang, H., Li, W., et al. (2013). A phase II study of oxaliplatin in combination with leucovorin and fluorouracil as first-line chemotherapy in patients with metastatic squamous cell carcinoma of esophagus. Cancer Chemother. Pharmacol. 71 (4), 905-911. doi: 10.1007/s00280-013-2081-5

Wang, Y., et al. (2019). Treatment-related adverse events of PD-1 and PD-L1 inhibitors in clinical trials: a systematic review and meta-analysis. JAMA Oncol. 5 (7), 1008-1019.
Weber, J. S., D’Angelo, S. P., Minor, D., Hodi, F. S., Gutzmer, R., Neyns, B., et al. (2015). Nivolumab versus chemotherapy in patients with advanced melanoma who progressed after anti-CTLA-4 treatment (CheckMate 037): a randomised, controlled, open-label, phase 3 trial. Lancet Oncol. 16 (4), 375-384. doi: 10.1016/S1470-2045(15)70076-8

Xu, R.H., Wang, F., Shi, J., Feng, J. F., Shen, L., Yang, S., et al. (2018). Recombinant humanized anti-PD-1 monoclonal antibody (JS001) as salvage treatment for advanced esophageal squamous cell carcinoma: preliminary results of an openlabel, multi-cohort, phase Ib/II clinical study. J. Clin. Oncol. 36 (4_suppl), 116116. doi: 10.1200/JCO.2018.36.4

Yarchoan, M., Johnson, B. A., Lutz, E. R., Laheru, D. A., and Jaffee, E. M. (2017). Targeting neoantigens to augment antitumour immunity. Nat. Rev. Cancer. 17, 209-222. doi: 10.1038/nrc.2017.74

Conflict of Interest: The authors declare that the research was conducted in the absence of any commercial or financial relationships that could be construed as a potential conflict of interest.

Copyright (c) 2019 Hong and Ding. This is an open-access article distributed under the terms of the Creative Commons Attribution License (CC BY). The use, distribution or reproduction in other forums is permitted, provided the original author(s) and the copyright owner(s) are credited and that the original publication in this journal is cited, in accordance with accepted academic practice. No use, distribution or reproduction is permitted which does not comply with these terms. 